\title{
Analysis of time to regulatory and ethical approval of SATVI TB vaccine trials in South Africa
}

\author{
H Geldenhuys, A Veldsman, M Tameris, A Luabeya, W Hanekom, H Mahomed, M Hatherill \\ South African Tuberculosis Vaccine Initiative (SATVI), Institute of Infectious Disease and Molecular Medicine (IIDMM); and School of Child and \\ Adolescent Health, Facutly of Health Sciences, University of Cape Town \\ H Geldenhuys, MB ChB, MFamMed \\ A Veldsman, N Nursing \\ M Tameris, $\mathrm{MB} \mathrm{ChB}$ \\ A Luabeya, MB ChB, MSc \\ W Hanekom, MB ChB, DCH, FCPaed \\ H Mahomed, MB ChB, MMed (Public Health) \\ M Hatherill, MD
}

Corresponding author: H Geldenhuys (hennie.geldenhuys@uct.ac.za)

\begin{abstract}
Background. Tuberculosis (TB) vaccine trials in South Africa must be approved by the Medicines Control Council (MCC) and by a human research ethics committee (HREC). Delays in regulatory and ethical approval may affect operational and budget planning and clinical development of the product.

Aim. Our aim was to analyse the time to regulatory and ethical approval for TB vaccine trials conducted by the South African Tuberculosis Vaccine Initiative (SATVI) and to evaluate factors that influence time to final approval.

Method. Sixteen new TB vaccine clinical trials conducted by SATVI between 2004 and 2012 on infants, children, and adults were included. The period between submission and final approval was determined for protocols submitted to the MCC and the University of Cape Town HREC. Results. Median approval time following first submission to the MCC was 122 days (IQR 112 - 168; range 71 - 350), and for protocol amendments 103 days (interquartile range (IQR) 76 - 141; range 23 - 191; $n=30$ ). Median time following first submission for HREC approval was 60 days (IQR 33 - 81; range 18 - 125), and for amendments 6 days (IQR 4 - 13; range 1 - 37; $n=30$ ). There was no significant difference in approval time by trial phase, year of submission, revisions required, study population, sample size, or whether a clinical research organisation (CRO) was used. Conclusion. The time needed for regulatory and ethics approval was highly variable, but MCC approval for first submissions took twice as long as HREC approval and was the primary determinant of time to final approval. National regulatory capacity should be strengthened to facilitate the conduct of new TB vaccine trials in this country with its high burden of TB.

S Afr Med J 2013;103(2):85-89. DOI:10.7196/SAMJ.6390
\end{abstract}

Regulatory and ethics review of clinical trial protocols is an important mechanism to ensure safe and ethical research on human participants. Such review processes are integral to the development of any new tuberculosis (TB) vaccine. Long delays in approval times may increase costs, complicate operational planning by sponsors and researchers, and lead to frustration and distrust in the system. Protracted regulatory processes can delay the development of critical new drugs and vaccines for high priority diseases such as TB and HIV. $^{2}$ Furthermore, lengthy delays in the approval of clinical trials might create the perception that a particular research environment is not conducive to investigational product development, with the risk that sponsors might choose to conduct the trials elsewhere.

Clinical trials of investigational products, including new candidate $\mathrm{TB}$ vaccines, must be pre-approved by a regulatory organisation and at least one human research ethics committee (HREC) in most parts of the world. The statutory regulatory organisation in South Africa (SA) is the Medicines Control Council (MCC). ${ }^{3}$

The South African Tuberculosis Vaccine Initiative (SATVI) is an academic research unit within the University of Cape Town (UCT) that has been involved in clinical TB research for over 10 years. ${ }^{4}$ SATVI has conducted 18 registration-standard vaccine trials, including clinical trials of Bacille Calmette-Guérin (BCG) vaccine and 5 different novel $\mathrm{TB}$ vaccine candidates. These studies have contributed to answering critical questions in novel $\mathrm{TB}$ vaccine development, including methods to measure the mycobacterial immune response and immune correlates of risk and protection against TB. ${ }^{5-7}$ As a result of this work, the SATVI clinical research site in Worcester, Western Cape Province, is widely regarded as the leading TB vaccine research site in the world. ${ }^{4}$

The objective of this study was to quantify our experience of the time taken to achieve regulatory and ethics approval for TB vaccine trials as a reflection of the SA research regulatory environment for $\mathrm{TB}$ vaccine research.

\section{Methods}

We determined the length of time for regulatory and ethics approval for all TB vaccine trials conducted at SATVI between 2004 and 2012 on the part of both the MCC and UCT Faculty of Health Sciences HREC. All applications were submitted simultaneously to both the MCC and HREC.

Approval time was defined as the time between date of protocol submission and the date on the letter confirming approval. In the case of the MCC, applications were delivered via courier to the MCC offices in Pretoria, and the date of receipt by the MCC was used as the submission date. Applications submitted to the UCT HREC were hand-delivered, and the date of delivery was used as the submission date. The MCC and HREC stipulate deadlines for applications prior to each committee meeting, and all applications met those deadlines. 
The regulatory and ethics submissions of all $\mathrm{TB}$ vaccine trial protocols conducted by SATVI since its first phase 1 trial of a novel TB vaccine in 2004 were included in this analysis. Data recorded for each protocol submission included whether this was a first application or a protocol amendment, the sponsor of the trial, the principal investigator (PI), phase of trial (I, II, III or IV), and the year and month of submission. Study population characteristics included the sample size and age group (infants, adolescents, adults). In certain instances, the application was submitted by SATVI in co-operation with the sponsor, and in other cases with the assistance of a clinical research organisation (CRO) in co-operation with SATVI and the sponsor. If the regulatory body or HREC requested a revised submission or further information before final approval, the number of protocol revisions required was recorded. In addition to the year of submission, approval time was compared before and after December 2008 to detect possible trends over time.

Data were analysed using Stata version 11 (Datacorp 2011). For MCC and HREC applications, median approval times were determined, with interquartile ranges (IQR) and minimummaximum ranges. Data were stratified according to review body (MCC or HREC) and whether this was a first or amendment protocol submission. A Kruskal-Wallis test was used for nonparametric data to compare approval times between MCC and HREC, and to determine whether approval time was affected by trial phase, year of submission, month of submission, revisions required, age of study population, sample size, or whether a CRO was used. Statistical significance was defined as a probability level of $\leq 0.05$.

\section{Results}

A total of 92 applications were submitted to the MCC and HREC for $16 \mathrm{~TB}$ vaccine trial protocols. Of these, 32 were for first applications (16 to MCC and 16 to HREC) and 60 (30 each to MCC and HREC) were for subsequent protocol amendments.

Median approval time for first submission to MCC was 122 days (IQR 112 - 168; range 71 - 350) (Figs 1 and 2). Approval time for amendment submissions to the MCC was shorter than for first submissions (median 103 days; IQR 76 - 141, range 23 - 191), but this difference was not statistically significant $(p=0.1)$. Two $(12 \%)$ of first submissions received MCC approval within 3 months, 6 (38\%) between 3 and 4 months, 3 (19\%) between 4 and 5 months, and 5 (31\%) longer than 5 months. Three (10\%) of amendment submissions

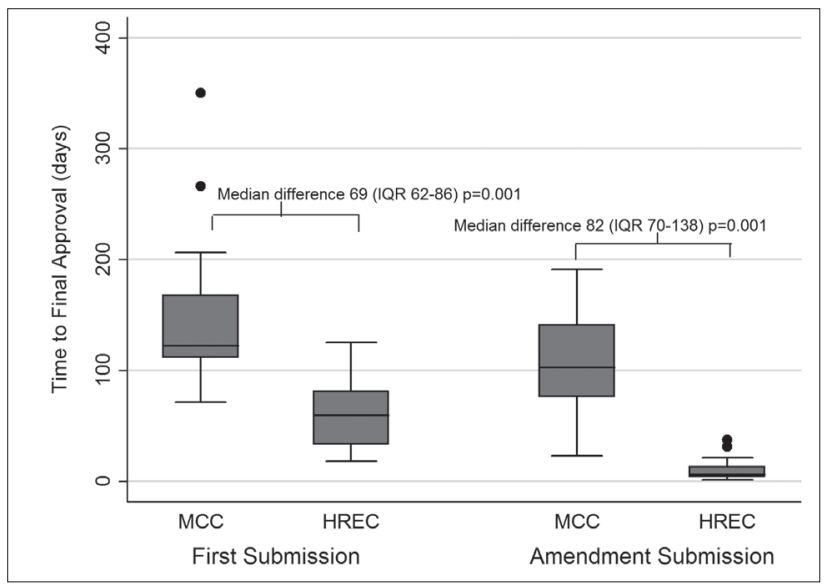

Fig. 1. Box-plot of approval times by first submissions and amendment submissions, and by regulatory body. MCC = Medicines Control Council of South Africa. HREC = University of Cape Town Human Health Sciences Faculty Research Ethics Committee.

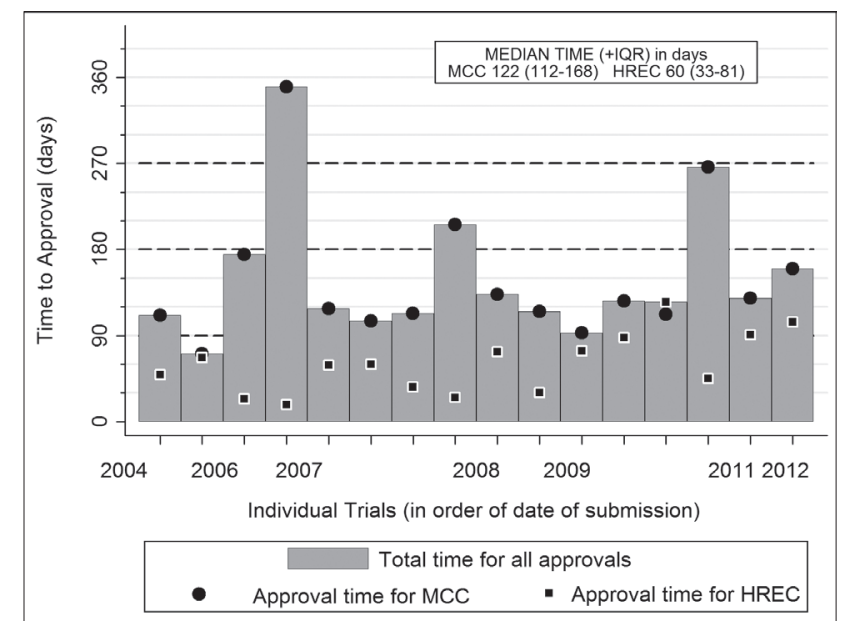

Fig. 2. Time to approval for first submissions per individual trial. Total time to final approval is shown, as well as individual times for MCC and HREC. MCC and HREC approval submissions were made simultaneously.

received MCC approval within 3 months, 18 (60\%) between 3 and 4 months, and $9(30 \%)$ longer than 4 months.

Median approval time for HREC first submissions was 60 days (IQR 33 - 81; range 18 - 125). For amendment submissions to HREC, this time was shorter (median 6 days; IQR 4 - 13; range 1 - 37; $p<0.001$ ) (Fig. 1). Four (25\%) first submissions were approved by HREC within 1 month, 5 (31\%) between 1 and 2 months, 5 (31\%) between 2 and 3 months, and 2 (13\%) between 3 and 4 months. For amendments, HREC approval was received in less than 1 month for 28 (93\%) submissions, and within 1 - 2 months in 2 (7\%) cases.

Median approval times for first and amendment submissions to the MCC were greater than for HREC ( $p=0.001)$ (Fig. 1). The median difference between MCC and HREC approval times was 69 days (IQR 62 - 86) for first submissions and 82 days (IQR 70 -138) for amendment submissions (Fig. 2).

There were no differences in approval time by clinical trial phase, year of submission, calendar month of submission, submission before or after December 2008, sponsor, PI, age of study population, sample size, or whether a clinical research organisation (CRO) was used, for either MCC or HREC submissions (Tables 1 and 2).

Of applications submitted to the MCC, $11 / 16$ (68\%) first submissions and 6/30 (20\%) amendment submissions required revision or additional information before final approval. The number of resubmissions required did not affect approval times by the MCC for first or amendment submissions. Thirteen (81\%) first HREC submissions and $1(3 \%)$ amendment submission required revision or additional information. HREC approval time for first submissions was not greater for submissions that required revision.

Three trials took longer than 6 months for initial approval (Table 2; Fig. 2). In all 3 cases, HREC approval had been given in less than 2 months. The reason for the longer MCC approval times for these particular trials was not known.

\section{Discussion}

We have described time to approval of TB vaccine trials by regulatory and ethics authorities and analysed the factors that influenced these approval times. MCC approval for first protocol submissions took twice as long as HREC approval and was the primary determinant of total time to final approval. There was considerable variability and unpredictability in the time required for the MCC approval process, which in the worst case took 12 months. HREC approved protocol 
Table 1. Approval time for MCC and HREC for first applications by protocol and submission factors $(N=16 \text { each })^{*}$

\begin{tabular}{|c|c|c|c|c|c|}
\hline & \multirow[b]{2}{*}{$N$} & \multicolumn{2}{|c|}{ MCC } & \multicolumn{2}{|c|}{ HREC } \\
\hline & & Median (IQR) & Range (min - max) & Median (IQR) & Range (min - max) \\
\hline Overall & 16 & $122(112-168)$ & $71-350$ & $60(33-81)$ & $18-125$ \\
\hline \multicolumn{6}{|l|}{ Phase of trial } \\
\hline Phase 1 & 2 & $130(126-133)$ & $126-133$ & $81(73-88)$ & $73-88$ \\
\hline Phase 2a & 12 & $116(108-168)$ & $71-350$ & $60(41-83)$ & $18-125$ \\
\hline Phase $2 b$ & 2 & $161(115-206)$ & $115-206$ & $28(25-30)$ & $25-30$ \\
\hline \multicolumn{6}{|l|}{$\mathrm{CRO}$} \\
\hline Yes & 12 & $117(108-152)$ & $71-350$ & $54(28-71)$ & $18-91$ \\
\hline No & 4 & $147(123-213)$ & $112-266$ & $89(59-115)$ & $45-125$ \\
\hline \multicolumn{6}{|c|}{ Age of study population ${ }^{\dagger}$} \\
\hline Infants & 7 & $129(112-206)$ & $105-350$ & $60(25-104)$ & $18-125$ \\
\hline Adolescents & 3 & $111(93-266)$ & $93-266$ & $49(45-74)$ & $45-74$ \\
\hline Adults & 6 & $122(113-133)$ & $71-175$ & $63(36-73)$ & $24-88$ \\
\hline \multicolumn{6}{|l|}{ Sample size } \\
\hline$<50$ & 9 & $113(105-126)$ & $71-350$ & $59(36-67)$ & $18-88$ \\
\hline $50-350$ & 5 & $133(129-160)$ & $112-266$ & $91(73-104)$ & $45-125$ \\
\hline$>1000$ & 2 & $161(115-206)$ & $115-206$ & $28(25-30)$ & $25-30$ \\
\hline \multicolumn{6}{|c|}{ Year of submission } \\
\hline $2004-2008$ & 9 & $118(111-175)$ & $71-350$ & $49(25-60)$ & $18-73$ \\
\hline $2009-2012$ & 7 & $126(112-160)$ & $93-266$ & $88(45-104)$ & $30-125$ \\
\hline \multicolumn{6}{|c|}{ Revised resubmission required } \\
\hline Yes & 10 & $131(111-175)$ & $71-350$ & $64(38-89)^{\ddagger}$ & $18-125$ \\
\hline No & 6 & $117(112-126)$ & $93-206$ & $43(30-61)$ & $24-73$ \\
\hline
\end{tabular}

amendments significantly more quickly than first submissions, but amendment approval time for the MCC was similar to first submissions.

To our knowledge, this is the first published analysis of regulatory approval times in the SA context for vaccine clinical trials. The South African Clinical Research Association (SACRA) has called for an online poll of research organisations to collect such data for all types of clinical trials on a wider scale, but these data have not yet been published. ${ }^{8}$

Time to approval by regulatory and ethics bodies may be affected by protocol factors, or by the capacity of the body to review and process the application. Our data suggest that approval time by the MCC is not significantly influenced by the complexity or safety risk associated with a particular protocol, such as the phase of trial, participant age group or sample size. The lack of correlation between approval time and protocol submissions that required clarification or resubmission is also noteworthy. It is likely, therefore, that capacity and administrative limitations are major drivers in approval delays by the MCC. The MCC has undergone structural changes and is committed to improving its systems and processes; ${ }^{3}$ although, in our experience, the trend in approval times did not improve over the 8 -year period represented by our data.

Time for approval of amendments was significantly shorter for the HREC than for the MCC. Amendments typically involve specific targeted changes to a protocol and do not require a full review of a protocol that has been submitted previously; this may be an area where the MCC could develop more efficient mechanisms.

Predictability and transparency of the review process is a key issue in regulatory and ethics approval, given that there was up to a five-fold variation in MCC approval times. Unpredictability of the approval process makes planning difficult for researchers and creates sponsor anxiety in the context of deadlines for grant funding or product development milestones. Greater transparency in the review process and a willingness to engage with investigators would improve stakeholder perceptions and create more confidence in the regulatory review system. For example, regulatory bodies such as the United States Food and Drug Administration (FDA) have made pro-active efforts to provide information about its ongoing performance on a continual basis, including backlogs in application approvals. ${ }^{9}$

Issues with regulatory capacity are not unique to $\mathrm{SA}$, although approval times in Europe and the USA tend to be shorter. The European Union (EU) adopted the Clinical Trials Directive (EUCTD) in 2001 to improve the clinical research process. ${ }^{10}$ The directive aimed to streamline approval process for all clinical trials by, inter alia, defining the role of central and local research ethics committees, allowing parallel and not sequential submissions to HREC and a national regulatory authority, and stipulating that authorities provide decisions within 60 days. In the United Kingdom (UK), a similar reform process for HREC approvals was instituted 


\section{RESEARCH}

Table 2. Summary of protocol characteristics for each TB vaccine trial submitted to MCC and HREC $(N=16)^{\star}$

\begin{tabular}{|c|c|c|c|c|c|c|c|c|}
\hline $\begin{array}{l}\text { Year of } \\
\text { submission }\end{array}$ & $\begin{array}{l}\text { Trial } \\
\text { phase }\end{array}$ & Study population & Vaccine type & $\begin{array}{l}\text { Sample } \\
\text { size, } N\end{array}$ & HIV status & $\begin{array}{l}\text { Latent TB } \\
\text { infection } \\
\text { status }\end{array}$ & $\begin{array}{l}\text { Time to } \\
\text { approval } \\
\text { MCC }\end{array}$ & $\begin{array}{l}\text { Time to } \\
\text { approval } \\
\text { HREC }\end{array}$ \\
\hline 2004 & $2 \mathrm{a}$ & $\begin{array}{l}\text { Mixed adults and } \\
\text { adolescents }\end{array}$ & $\begin{array}{l}\text { Viral-vectored GMO } \\
\text { (vaccinia virus) }\end{array}$ & 36 & Negative & Negative & 111 & 49 \\
\hline 2006 & $2 \mathrm{a}$ & Adults & $\begin{array}{l}\text { Viral-vectored GMO } \\
\text { (vaccinia virus) }\end{array}$ & 48 & Mixed & Mixed & 71 & 67 \\
\hline 2006 & $2 a$ & Adults & $\begin{array}{l}\text { Viral-vectored GMO } \\
\text { (adenovirus) }\end{array}$ & 40 & Negative & Negative & 175 & 24 \\
\hline 2007 & $2 \mathrm{a}$ & Infants and children & $\begin{array}{l}\text { Viral-vectored GMO } \\
\text { (vaccinia virus) }\end{array}$ & 168 & Negative & Negative & 350 & 18 \\
\hline 2007 & $2 \mathrm{a}$ & Adults & $\begin{array}{l}\text { Fusion protein sub-unit } \\
+ \text { adjuvant }\end{array}$ & 45 & Negative & Mixed & 126 & 59 \\
\hline 2008 & $2 b$ & Infants & $\begin{array}{l}\text { Viral-vectored GMO } \\
\text { (vaccinia virus) }\end{array}$ & 2784 & Negative & Negative & 206 & 25 \\
\hline 2008 & $2 \mathrm{a}$ & Infants & $\begin{array}{l}\text { Viral-vectored GMO } \\
\text { (adenovirus) }\end{array}$ & 54 & Negative & Negative & 105 & 60 \\
\hline 2008 & $2 \mathrm{a}$ & Adults & $\begin{array}{l}\text { Fusion protein sub-unit } \\
+ \text { adjuvant }\end{array}$ & 40 & Negative & Negative & 113 & 36 \\
\hline 2008 & 1 & Adults & $\begin{array}{l}\text { Live attenuated } \\
\text { BCG with isoniazid } \\
\text { pretreatment }\end{array}$ & 82 & Negative & Positive & 133 & 73 \\
\hline 2009 & $2 \mathrm{a}$ & Adolescents & $\begin{array}{l}\text { Fusion protein sub-unit } \\
+ \text { adjuvant }\end{array}$ & 60 & Negative & Mixed & 93 & 74 \\
\hline 2009 & $2 b$ & Infants & $\begin{array}{l}\text { Viral-vectored GMO } \\
\text { (adenovirus) }\end{array}$ & $4192^{\dagger}$ & Negative & Negative & 115 & 91 \\
\hline 2011 & 1 & Adults & $\begin{array}{l}\text { Fusion protein sub-unit } \\
+ \text { adjuvant }\end{array}$ & 25 & Negative & Mixed & 126 & 88 \\
\hline 2011 & $2 a$ & $\begin{array}{l}\text { Mixed adults, } \\
\text { adolescents and infants }\end{array}$ & $\begin{array}{l}\text { Viral-vectored GMO } \\
\text { (vaccinia virus) }\end{array}$ & 112 & Mixed & Mixed & 112 & 125 \\
\hline 2011 & $2 \mathrm{a}$ & Adolescents & $\begin{array}{l}\text { Fusion protein sub-unit } \\
+ \text { adjuvant }\end{array}$ & 240 & Negative & Negative & 266 & 45 \\
\hline 2011 & $2 a$ & Infants & $\begin{array}{l}\text { Viral-vectored GMO } \\
\text { (vaccinia virus) }\end{array}$ & 340 & $\begin{array}{l}\text { HIV } \\
\text { exposed }\end{array}$ & Negative & 129 & 91 \\
\hline 2012 & $2 a$ & $\begin{array}{l}\text { Mixed adults and } \\
\text { infants }\end{array}$ & $\begin{array}{l}\text { BCG with novel } \\
\text { administration device }\end{array}$ & 96 & Negative & Negative & 160 & 104 \\
\hline
\end{tabular}

by the Central Office for Research Ethics Committees (COREC) in $2007 .{ }^{11}$ In practice, these measures are not always effective, and end users of the system have expressed concern over inefficiencies. ${ }^{11,12}$ Nevertheless, compared with our SA experience, approval times for all types of clinical trials in the EU and UK are shorter (reported mean first approval times range from 43 to 75 days). ${ }^{10-13}$ In the USA, new products, including vaccine candidates, need to be approved as part of the Investigational New Drug approval process of the FDA before being approved by ethics committees for testing in clinical trials. Reported median approval times for all trials are significantly shorter in the USA (15 - 45 days). ${ }^{10}$ However, in other parts of Africa and the developing world, little has been published on regulatory approval times. Anecdotal evidence from our collaborating TB vaccine trial sites in Uganda and Mozambique indicate that approval times are longer - approximately 5 months for local and national HREC approval, and an additional $8-18$ months for national authority approval (E Wobudeya and J Sacralal, 2012, personal communications). Therefore, it is noteworthy that SA regulatory approval times are appreciably shorter than in other developing countries.

This study is limited by a small sample size and therefore we were unable to perform meaningful sub-analysis of less common factors that might substantially affect approval times. Our analysis does create a useful platform for discussion, and invites other research sites and organisations to share their experiences. The fact that our analysis includes data from a single site and for a single group of 


\section{RESEARCH}

investigational products (TB vaccines) is a particular strength, since our analysis naturally controls for heterogeneity in research site and investigational product.

In conclusion, we found that variability in regulatory and ethics approval, particularly by the MCC, is a significant factor in the SA clinical trial environment. Prolonged regulatory delays may impede the conduct of research in critical areas such as TB, and limit the ability of SA researchers to compete in the international arena. We acknowledge that the MCC is engaged in a process to optimise the regulatory pathway. We recommend that stakeholders in academia, industry and government engage in discussion to support the MCC in its planned restructuring. Furthermore, we call for state investment and capacity building for African regulatory bodies, to facilitate $\mathrm{TB}$ vaccine research in high TB burden countries where it is most needed.

\section{References}

1. Bollyky TJ, Cockburn IM, Berndt E. Bridging the gap: improving clinical development and the regulatory pathways for health products for neglected diseases. Clin Trials 2010;7(6):719-734. [http:/ dx.doi.org/10.1177/1740774510386390

2. Jindani A, Griffin GE. Challenges to the development of new drugs and regimens for tuberculosis. Tuberculosis 2010;90(3):168-170. [http://dx.doi.org/10.1016/j.tube.2010.03.006]
3. Baird D van Niekerk D. The regulation of clinical trials in South Africa. Qual Assur J 2004:8:33-36. [http:///x doi.org/10.1002/qaj. 252

4. Hanekom WA, Hawkridge A, Mahomed H, et al. SATVI - after 10 years closing in on a new and better vaccine to prevent tuberculosis. S Afr Med J 2012;102(6):438-441.

5. Kagina BM, Abel B, Scriba TJ, et al. Specific T cell frequency and cytokine expression profile do not correlate with protection against tuberculosis after bacillus Calmette-Guerin vaccinatio of newborns. Am J Resper Crit Care Med 2010,182(8):1073-1079. [http://dx.doi.org/10.116 rccm.201003-0334OC]

6. Soares AP, Scriba TJ, Joseph S, et al. Bacillus Calmette-Guerin vaccination of human newborns induces T cells with complex cytokine and phenotypic profiles. J Immunol 2008;180(5):3569-3577.

7. Scriba TJ, Kalsdorf B, Abrahams DA, et al. Distinct, specific IL-17- and IL-22-producing CD4+ T cell subsets contribute to the human anti-mycobacterial immune response. J Immunol 2008;180(3):19621970.

8. South African Clinical Research Association home page. www.sacraza.com (accessed 13 September 2012)

9. Asamoah AK, Sharfstein JM. Transparency at the Food and Drug Administration. N Engl J Med 2010;362(25):2341-2343. [http://dx.doi.org/10.1056/NEJMp1005202]

0. Heerspink HJ, Dobre D, Hillege HL, Grobbee DE, De Zeeuw D. Does the European Clinical Trials Directive really improve clinical trial approval time? Br J Clin Pharmacol 2008;66(4):546-550.

1. Mallick AA, O'Callaghan FJ. Research governance delays for a multicentre non-interventional study. R Soc Med 2009;102(5):195-198. [http://dx.doi.org/10.1258/jrsm.2009.080397]

12. Salman RA, Dennis MS, Sandercock PAG, White PM, Warlow C. Research governance impediments to clinical trials: a retrospective survey. J R Soc Med 2007;100:101-104. [http://dx.doi.org/10.1258 jrsm.100.2.101

13. Chester P, Alaam S, Cooper P, Risley C, Dornhorst A. Local clinical trial approval times in the UK. International Journal of Pharmaceutical Medicine3 2006;20(5):317-20. [http://dx.dol org/10.2165/00124363-200620050-00006

Accepted 23 October 2012 